
the Exposure Buildup Factors of Low-Energy Gamma Rays

H. HIRAYAMA, Y. NAMITO and S. BAN

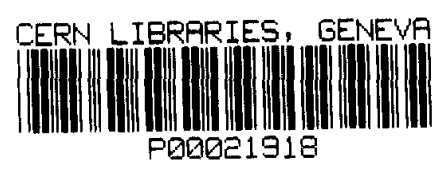

Submitted to the 8th International Conference on Radiation Shielding, Arlington, TX, U.S.A., April 24 - 28, 1994. 


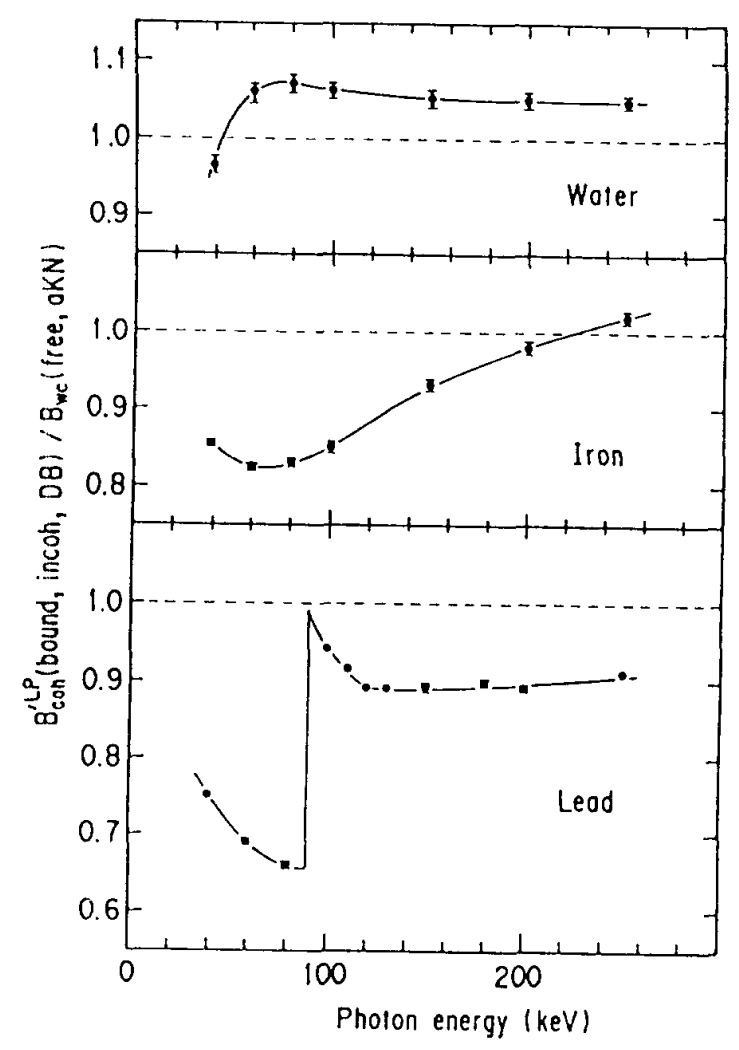

Fig.4. Ratio of pseudo exposure buildup factors $x$ th coherent scattering and with Compton scatternis while taking into account the binding effect and Joppler broadening applying linear polarization for whth coherent and incoherent scattering to the true mes without coherent and free electron scattering at $210-m f p$ penetration depth as a function of the photon anergy for water, iron, and lead. 
National Laboratory for High Energy Physics, 1994

KEK Reports are available from:

Technical Information \& Library

National Laboratory for High Energy Physics

1-1 Oho, Tsukuba-shi

Ibaraki-ken, 305

JAPAN

Phone: $\quad 0298-64-1171$

Telex: $\quad 3652-534$ (Domestic)

(0)3652-534 (International)

Fax: $\quad$ 0298-64-4604

Cable: $\quad$ KEK OHO

E-mail: LIBRARY@JPNKEKVX (Bitnet Address)

library@kekvax.kek.jp (Internet Address) 


\section{EFFECTS OF IINEAR POLARIZATION AND DOPPLER BROADENING ON THE EXPOSURE BUILDUP FACTORS OF IOW-ENERGY GAMMA RAYS}

\author{
Iirleo Hirayama \\ National Laboratory for \\ High Energy Pliysics \\ 1-1, Oho, Tsukuba-shi \\ Ibaraki, 305 .Japan \\ $81-(298)-64-5493$
}

\author{
Yoshihito Namito \\ National Laboratory for \\ High Energy Physics \\ 1-1, Oho, Tsukuba-shi \\ Ibaraki, 305 Japan \\ $81-(298)-64-5497$
}

\author{
Syuichi Ban \\ National Laboratory for \\ High Energy Physics \\ 1-1, Oho, Tsukuba-shi \\ Ibaraki, 305 Japan \\ $81-(298)-64-5491$
}

\begin{abstract}
ABSTRAC'T
The effects of including linear polarization and Doppler broadening in a ralculation of the exposure buildup factors for plane normal gamma-ray sources have been investigated by using an improved electron gamma shower Monte Calro code, EGS4, for water, iron, and learl in the 40- to $250-\mathrm{keV}$ range for penetration depths of up to $16 \mathrm{mfp}$. The "psendo" exposure buildup factors were calculated in order to determine these effects combined with the effects of coherent amd/or incoherent scattering.

The psendo exposure buildup factor increases upon including linear polarization and decreases upon including Doppler broadening. The degree of each effect varies with the atomic number of the material. The effect of linear polarization is large for materials of smal] atomic mumber; that of the Doppler broadening is large for materials of medium and large atomic number.
\end{abstract}

\section{INTRODUCTION}

The effects of including coherent scattering and the binding effect for Compton scattering (incoherent scattering) in a calculation of the exposure buildup factors for plane normal gamma-ray sources had been investigated' by one of the authors, and are citer in ANSI/ANS-6.4.3, ${ }^{2}$ a new buildup factor compilation. In the investigation discussed in the reference 1 , however, the incolerent scattering cross section, including the binding correction, were applied for only the total cross section. The angular distribution of incoherent scattering is also affecter by the binding effect and must be taken into arcount. This was studied by Angelopoulos et al." for only water. Doppler broadening upon incoherent scattering is also supposed to contribute to the buildup factors of low-energy photons. Moreover, linear polarization at coherent and incoherent scattering may affect photon transport in the medium.

The object of the present study was to investigate the effect of linear polarization at both coherent and incoherent and incoherent scattering, including the angular distribution and Doppler broadening upon exposure buildup factors in the 40- to 250 -keV range for typical shielding materials, such as water, iron and lead. The gamma-ray sources considered were plane normal to the material. The exposure buildup factor is based on the encrgy absorption response of air; that is, exposure is assumed to be equivalent to the albsorbed dose in air, as measured by a nonperturbing retector.

\section{DEFINITION OF THE BUILDUP FAC- TOR.}

Builulup factor data are customarily presented as a function of the distance measured in the meanfree-paths. The mean-free-path, in turn, can vary for a given linear distance (e.g., in centimeters) or mass per unit area thickness, depending on how the mass attenuation coefficient is defined, e.g., with or without coherent scattering, and also whether or not electron bincling for Compton scattering is taken into account. The mean-free-path in the new standard data is for no coherent scattering and without considering any electron binding effect for Compton scattering. It would be desirable to determine the size of the error due to neglection of various reactions in the transport calculations at the same distance. For this purpose, a "pseudo" exposure buildup factor (defined in reference 1) was used (definition 2). Various pseudo exposure buildup factors used in this paper are expressed as follows: 


$$
\begin{aligned}
& I_{i n h}^{\prime}(f \operatorname{rec}, a K N)= \\
& \text { b).oh }(\text { frec, } a K N: x) / D_{0} \exp \left(-\mu_{t} x\right) \text {, } \\
& l^{\prime} \quad P_{h}^{\prime \prime}(\operatorname{lrec}, a K N)= \\
& D^{l, l}(\text { free, } a K N: x) / D_{0} \exp \left(-\mu_{1} x\right) \text {, } \\
& f:(b o m n d, a K N)= \\
& \left.D_{w r} \text { (bound, aKN : } x\right) / D_{0} \exp \left(-\mu_{t} x\right) \text {, } \\
& \text { I!' (bonend, incoh })= \\
& D_{\text {we }}(\text { bound, incoh : } x) / D_{0} \exp \left(-\mu_{t} x\right) \text {, } \\
& \text { ' } \\
& D_{\text {we }}(\text { bound, incol, } D B: x) / D_{0} \exp \left(-\mu_{t} x\right) \text {, } \\
& \text { I' i, } \left.{ }^{\prime} \text { (bound, incoh, } D B\right)= \\
& D_{\text {coh }}^{L P}(\text { bound, incoh, } D B: x) / D_{0} \exp \left(-\mu_{t} x\right) \text {, }
\end{aligned}
$$

$\therefore o^{\prime}$ : with coherent scattering both for total scattering and angular distribution,

w.: : without coherent scattering,

frec: free-electron Compton scattering cross section for total Compton scattering,

bound: incoherent scattering cross section for total Compton scattering:

$" K N$ : angular distribution based on the freeelectron Klein-Nishina cross section for Compton scattering,

incoh: angular distribution including the binding eflect for Compton scattering,

DE: include Doppler broadening for Compton scat tering,

II': include linear polarization in the scattering,

B: psendo exposure buildup factor,

$L^{\prime}$ : exposure at $x$ centimeters,

$D_{1}$ : exposiue for source photon,

$H_{t}$ : linear attenuation coefficient with free electron Compton scattering and without coherent srattering $\left(\mathrm{cm}^{-1}\right)$.

'Therefore, $B_{\text {coh }}^{\prime \prime \prime}($ bound, incoh, $D B)$ is the pseuds: waowe buildup factor with coherent scattering and wita Compton scattering, while taking account of the simling effect and Doppler broadening, applying linear warization both for coherent and incoherent scatterne. Using the same notation, the standard exposure mildup factor can be expressed as

$$
\begin{aligned}
& B_{m .}(\text { free, } a K N)= \\
& \quad D_{w c}(\text { frec }, a K N: x) / D_{0} \exp \left(-\mu_{1} x\right) .
\end{aligned}
$$

The treatment of each phenomenum of coherent au. Compton scattering on each exposure and pseudo "xposure buildup faclors are summarized in Table $\mathrm{I}$.

\section{METHOD OF CALCULATION}

For simulating photon transport in the medium, a general electromagnetic radiation transport code, Electron Gamma Shower Version 4 (EGS4), ${ }^{1}$ was used. The EGS4 calculations were made in one-dimensional plane. The data $u_{\mathrm{p}}$ to $16 \mathrm{mf}$ ) were estimated from calculations for a 18 -mfp-thick semi-infinite plane. The linear attenuation coefficient without coherent scattering and without considering any electron binding effect for Compton scattering was used to determine the mean-free-path. Table II gives the linear attenuation coefficients with and without coherent scattering and considering the electron binding effect for Compton scattering at the source energies and the material densities used in the calculation. Linear polarization and Doppler broadening were implemented into the EGS4 system by the authors ${ }^{5,6}$ and applied to this work.

In the EGS4 calculation, the exposure was obtained from the photon flux and the energy transfer coefficient of air. The pseudo exposure buildup factor was calculated by dividing the exposure calculated by EGS4 by the exposure of the analytically calculated unscattered photons, which was calculated using the linear attenuation coefficient without coherent scattering and without considering the electron binding effect for Compton scattering. An exponential transform and next event surface crossing technicues are userl to obtain sufficient. statistics at deep penetration.

The photoelectric reaction and coherent scattering cross section were taken from a compilation by Storm and Israel. ${ }^{7}$ The mass energy transfer coefficients of air were taken from Hubbell's compilation. ${ }^{8}$

\section{RESULTS AND DISCUSSION}

The ratios of the various pseudo exposure buildup factors to the true one without coherent and free electron Compton scattering, $B_{1 v c}($ free, $a K N)$, for water, iron, and lead at $40,60,100$ and $200 \mathrm{keV}$ are given in Figs. 1, 2 and 3, respectively. These figures clearly show each effect on the psendo exposure buildup factors and their energy and $\mathrm{Z}$ dependence.

The pseudo exposure buildup factor increases with the inclusion of the electron binding correction to Compton scattering for the total cross section; its increasing rate is decreased upon including the angular distribution based on the incoherent scattering function due to suppression of scattering in the forward direction. This tendency is remarkable for water and is larger for lower energy photons. In the case of iron and learl, the same tendency is smaller than that for water, and the effect is larger for higher energy photons. Compton-scattered photon energy is broadened by a 
pre-collision motion of elertirn, i.e., Doppler broadening. The inclusion of this Doppler broarlening changes the photon energy after Compton scattering at a fixed angle. The degree of hroarlening is largex for high- $Z$ material. The effect to a pseudo exposure buildup depends on the change in the total and the scattering cross section in the expanded energy region. In the case of water, the effect of Doppler broadening is very small at all energies due to the small broadening. Except for the rase of iron at $40 \mathrm{keV}$, the inclusion of Doppler broadening decreases the pseudo exposure buildup factor.

The effert of coherent scattering, which decreases the pseudo exposure buildup factor, and is remarkable for a high-Z material, is slightly recovered by including linear polarization. For deep penetrations, the inclusion of polarization effects in a calculation increases the predicted photon fhux, due to favoring of roplanar scattering. This effect occurs only when a photon is scaltered several times in the medium and, therefore, can be seen in water only.

As the net effect, the inclusion of angular distribution and doppler broadening at incoherent scattering and that of linear polarization for both coherent and incoherent scaltering decreases the rate of any overestimated or underestimated tendency dine to including incoherent and colerent. scattering in the total cross section, respectively. The energy dependence of the net effect at $10 \mathrm{mfp}$ is shown in Fig. 4. In the case of rater, the net effect increases the psendo exposure buildup factors, except at $40 \mathrm{keV}$; its rate is less than $7 \%$ for all of the energy region consirlered. The net effoct always has a recreasing tendency in the case of leat, and is most remarkable just below the K-edge, because the contribution of coherent scattering is maximum at that energy. In the case of iron, the pseudo exposure buildup factor increases with an increase of the photon energy, except at $40 \mathrm{keV}$.

\section{SUMM $\wedge R Y$}

The pseudo exposure buiflup factors, with linearly polarization for both incoherent and coherent scattering and Doppler broadening at incoherent scattering in water, iron, and leart were calculated for plane normally incident sources of 40 t.e $250 \mathrm{keV}$. The pseurlo exposure buildup factors obtained give correction factors to the standard data, i.e. $B_{\text {r.m. }}^{\prime \prime}($ bound, incoh, $D B) / B_{\text {w. }}($ frec,$a K N)$.

The present study reveals that linearly polarization and Doppler broarlening also affect the psendo exposure buildup factor. The inclusion of angular distribution and Doppler broadening at incoherent scattering decreases the pseudo exposure buildup factor, and that of linear polarization increases it. The effect of Doppler broadening is remarkable for iron and lead and that of linearly polarization is seen only for water in the energy region studied.

All calculations were performed using HITACHI's Unix workstations (3050RX) at the Data Handling Center of National Laboratory for High Energy Physics (KEK).

\section{REFERENCES}

1. H. Hirayama and D.K.Trubey, "Effects of Incoherent and Coherent Srattering on the Exposure Buildup Factors of Low-Energy Gamma Rays", Nucl. Sci. Eng., 99,145-156 (1988).

2. American National Standard Gamma-Ray Attenuation. Coefficients and Buildup Factors for Enginecering Matcrial ANSI/ANS-6.4.3-1991, American Nuclear Society (1992).

3. A. Angeloponlos, A. Perris, K. Sakellarious, L. Sakelliou, K. Sarigiannis and G. Zarris, "Accurate Monte Carlo calculations of the combined attenuation and build-up factors, for energies (20$1500 \mathrm{keV}$ ) and distances $(0-10 \mathrm{~cm})$ relevant in branchytherapy", Phys. Mcd. Binl., 36, 763-778 (1991)

4. W. R. Nelson, H. Hirayama and D. W. O. Rogers, The EGS4 Code System, SLAC-265, Stanford Linear Accelerator Center (1985).

5. Y. Namito, S. Ban and II. Hirayama, "Implementation of linearly-polarized photon scattering into the EGS4 Code", Nucl. In.str. and Meth., A332, $277-283$ (1993).

6. Y. Namito, S. Ban and H. Hirayama, "Implementation of Doppler Broadening in to the EGS4 Code", to be submitted to Nucl. In.str. and Meth.

7. E. Storm and H. I. Israel,"Photon Cross Sertion from $1 \mathrm{keV}$ to $100 \mathrm{MeV}$ for Elements $\mathrm{Z}=1$ to $Z=100 "$ ", At. Data Nucl. Data Tables, 7, 565 $(1970)$.

8. J. Hubbell, Photon Cross Sections, Attemuation Cocfficients, and Energy Absorption Coefficicnts from 10 keV to $100 \mathrm{GeV}$, NSRDS-NBS 29, National Bureau of Standards (1069). 
Table I

Treatment of coherent and Compton scattering in exposure and pseurlo exposure buildup factor

\begin{tabular}{|c|c|c|c|c|c|c|c|}
\hline & \multicolumn{3}{|c|}{ Coherent scattering } & \multicolumn{4}{|c|}{ Compton scattering } \\
\hline & total & angular & $\mathrm{LP}$ & total & angular & $\overline{\mathrm{DB}}$ & LP \\
\hline$B_{w c}($ free,$a K N)$ & $x$ & $x$ & $x$ & free & $a \bar{K} N$ & $x$ & $x$ \\
\hline$B_{c o h}^{\prime}($ free,$a K N)$ & 0 & 0 & $x$ & free & $a K N$ & $x$ & $x$ \\
\hline$B_{\text {coh }}^{I I P}($ free, $a K N)$ & 0 & 0 & 0 & free & $a K N$ & $x$ & 0 \\
\hline$B_{u c}^{\prime}($ bound, $a K N)$ & $x$ & $x$ & $x$ & bonind & $a K N$ & $x$ & $x$ \\
\hline$B_{u j c}^{\prime}($ bound, incoh $)$ & $x$ & $x$ & $x$ & bontnd & incoh & $x$ & $x$ \\
\hline$B_{u, c}^{\prime}($ boumd, incoh, $D B)$ & $x$ & $x$ & $x$ & bound & incoh & 0 & $x$ \\
\hline$B_{\text {coh }}^{\text {ld }}($ bound, incoh, $D B)$ & 0 & $\mathrm{O}$ & $\mathrm{O}$ & bonend & incoh & 0 & 0 \\
\hline
\end{tabular}

Table II

Linear Altenuation Coeflicients and Material Densities

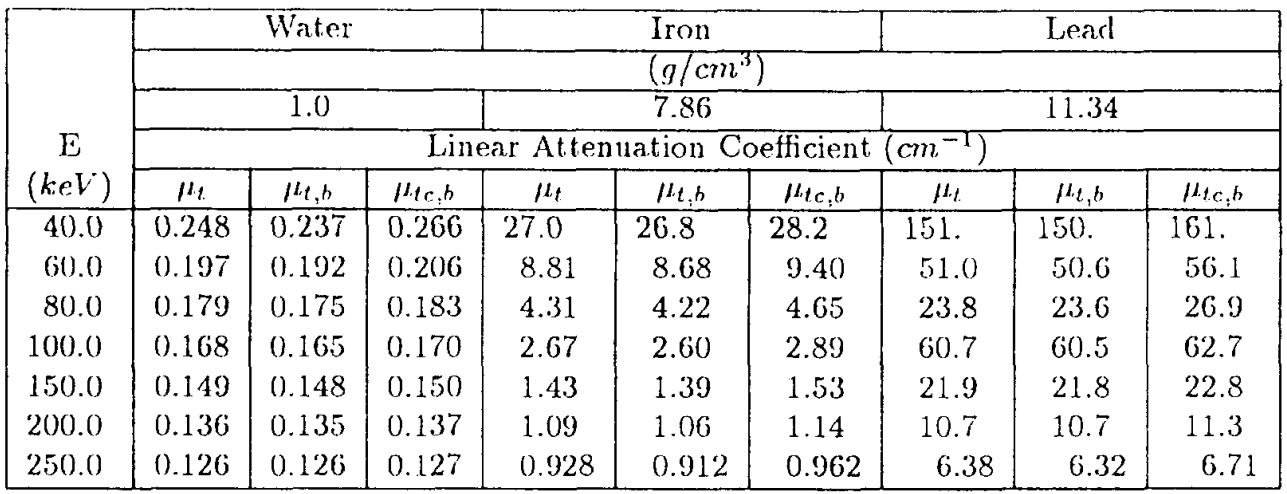

$\mu_{i}$ : Linear attenuation coefficient using a free electron Compton scattering cross section without coherent scattering.

$\mu_{t, b}$ : Linear attenuation coefficient using a bound electron Compton scattering cross section without coherent scattering.

$\mu_{t r, 1,}$ : Linear attenuation coefficient using a bound electron Compton scattering cross section with coherent scatteririg. 

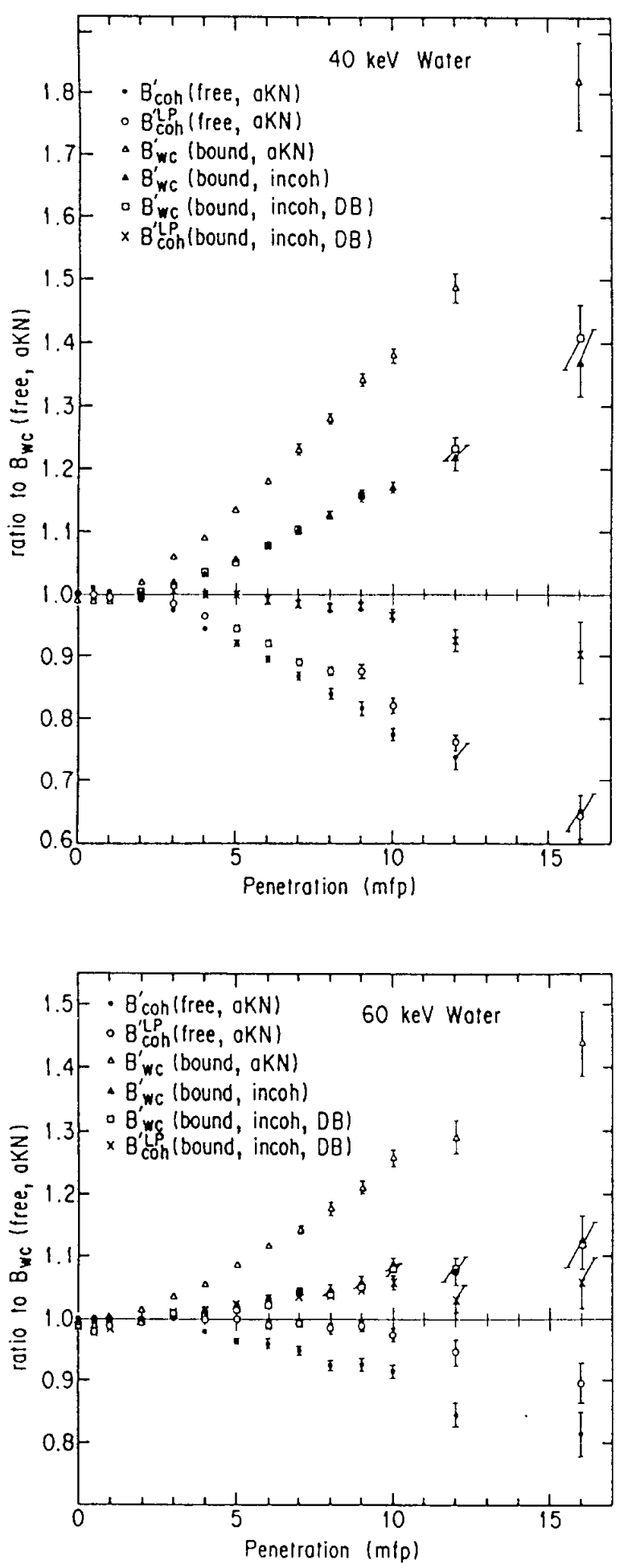
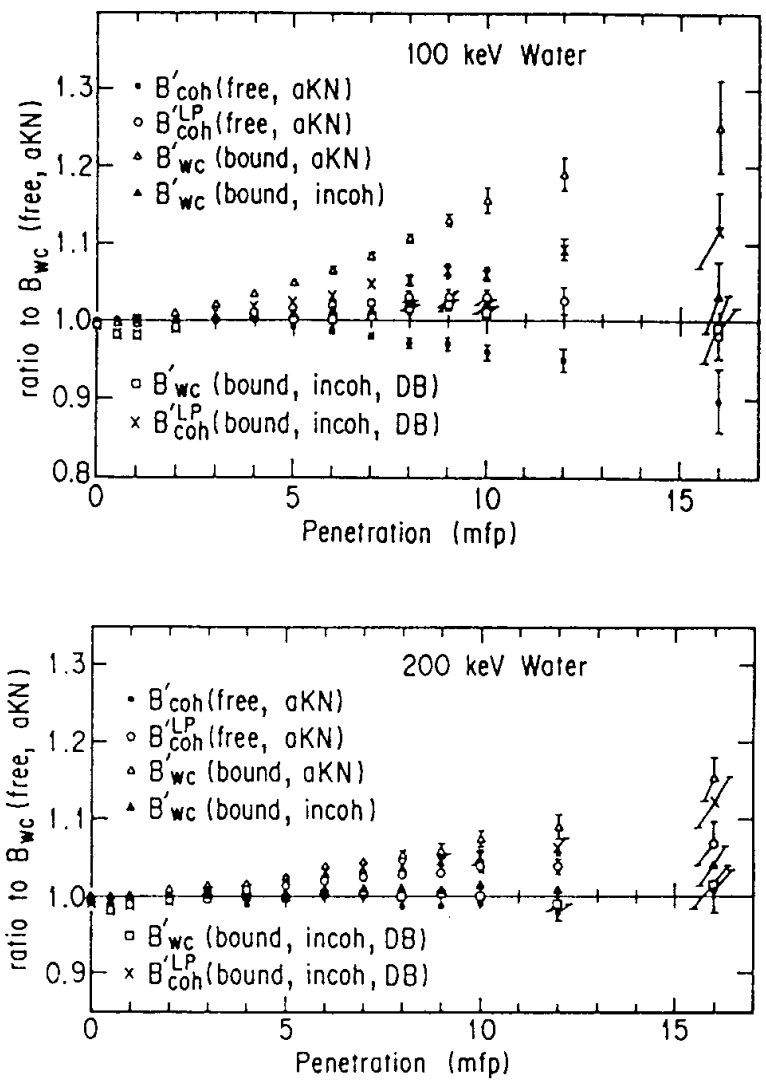

Fig.l. Ratio of various pseudlo exposure buildup factors to the true ones without coherent and free electron scattering for water at 40,60, 100, and $200 \mathrm{keV}$. 

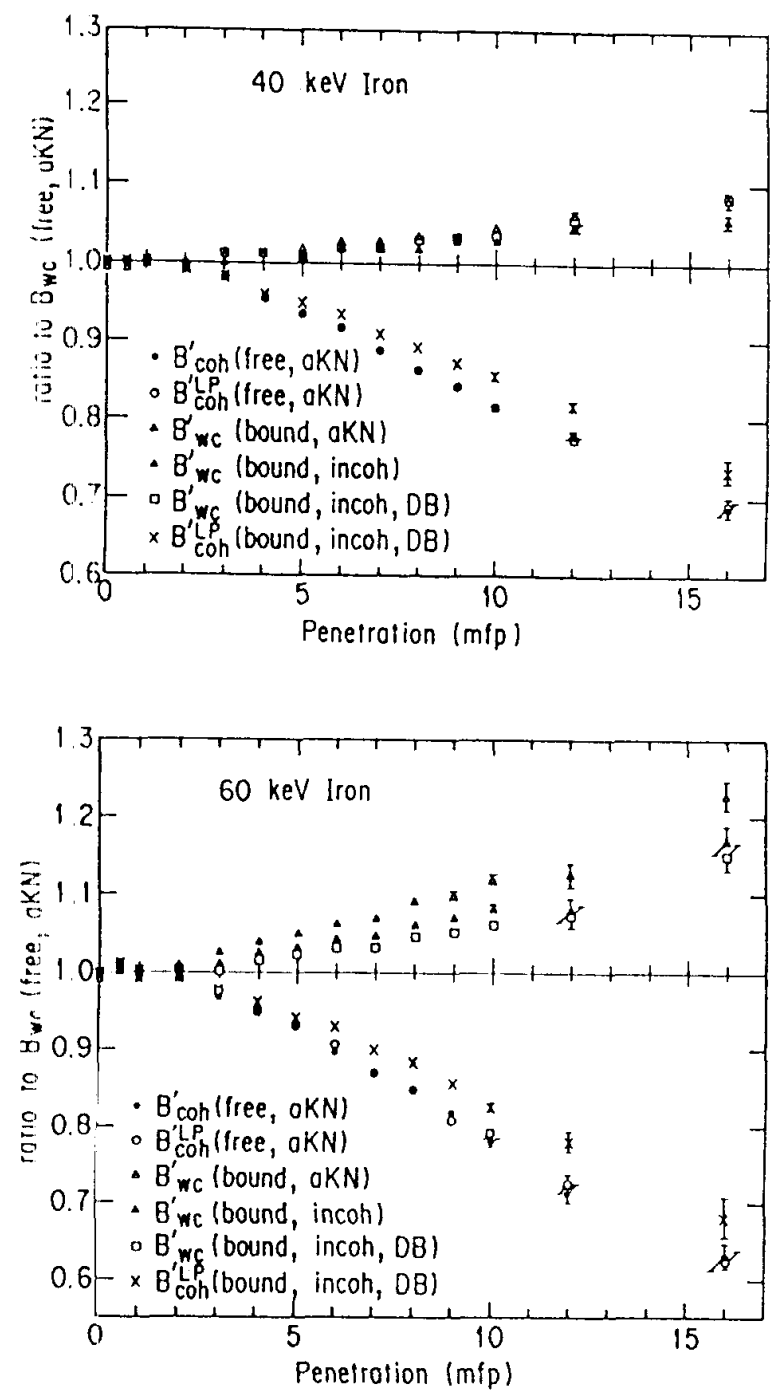


Fig.2. Ratio of various psendo exposure buildup factors to the true ones without coherent and free electron cittiering for iron at 40 , 60,100 , and $200 \mathrm{keV}$. 

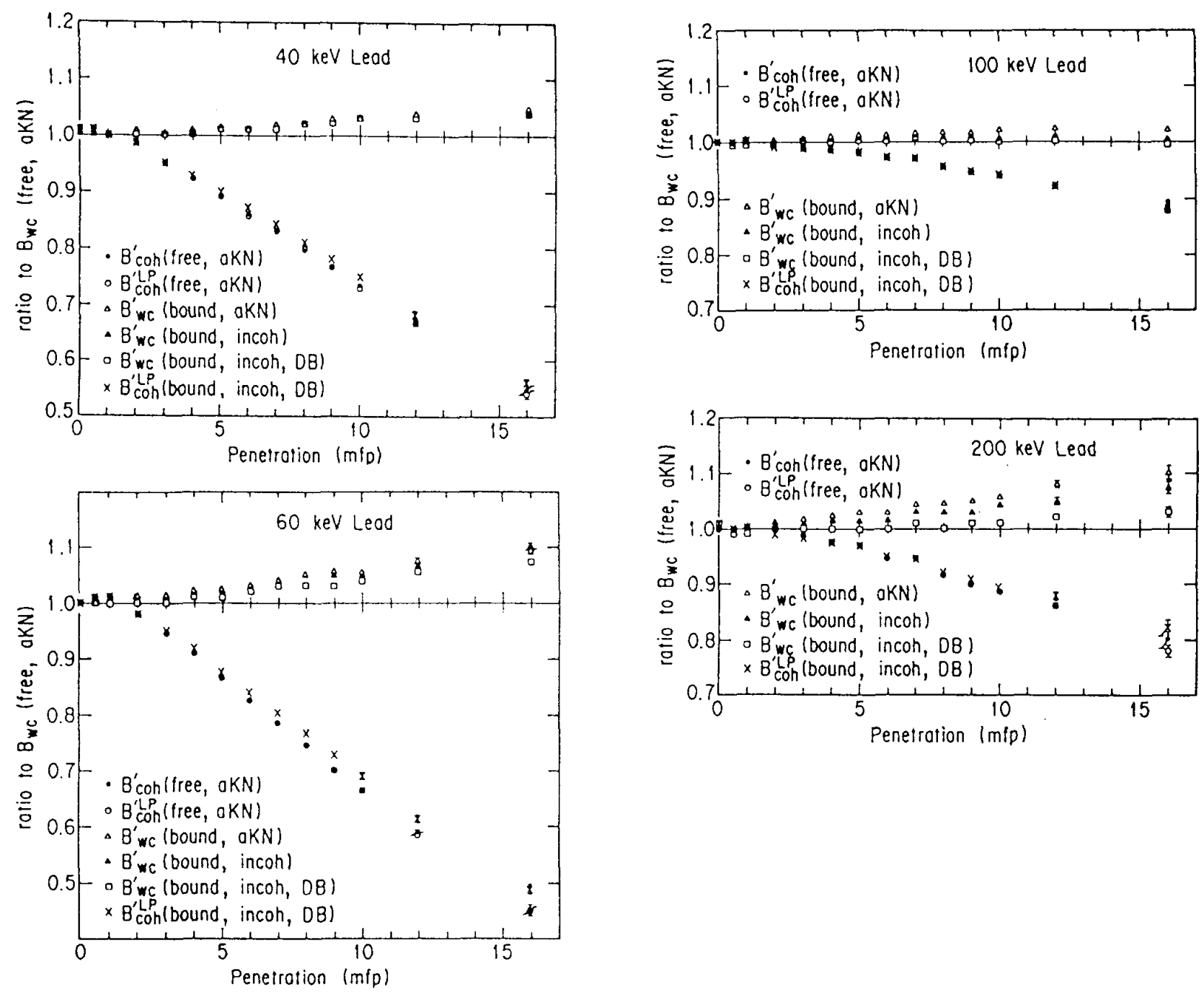

Fig.3. Ratio of various psendo cxposure builup factors to the true ones without coherent and free electron scattering for lead at $40,60,100$, and 200$) \mathrm{kov}$. 